\title{
One-loop Effective Action for a Generic 2D Dilaton Gravity Theory
}

\author{
Aleksandar Miković 目目目 \\ Departamento de Fisica Teórica and IFIC, Centro Mixto Universidad de \\ Valencia-CSIC, Facultad de Fisica, Burjassot-46100, Valencia, Spain \\ Voja Radovanović $⿴$ \\ Faculty of Physics, P.O. Box 368, 11001 Belgrade, Yugoslavia
}

\begin{abstract}
We study the one-loop effective action for a generic two-dimensional dilaton gravity theory conformally coupled to $N$ matter fields. We obtain an explicit expression for the effective action in the weak-coupling limit under a suitable restriction of the dilaton potential asymptotics. Our result applies to the CGHS model as well as to the spherically symmetric general relativity. The effective action is obtained by using the background-field method, and we take into account the loop contributions from all the fields in the classical action and from the ghosts. In the large- $N$ limit, and after an appropriate field redefinition, the one-loop correction takes the form of the Polyakov-Liouville action.
\end{abstract}

\footnotetext{
${ }^{1}$ On leave from Institute of Physics, P.O. Box 57, 11001 Belgrade, Yugoslavia

${ }^{2}$ Supported by Comissión Interministerial de Ciencia y Tecnologia

${ }^{3}$ E-mail: mikovic@lie1.ific.uv.es

${ }^{4}$ E-mail: rvoja@rudjer.ff.bg.ac.yu
} 


\section{Introduction}

Two-dimensional (2d) dilaton gravity theories are useful models for gaining understanding about the quantum properties of black holes [1]. A usual way of exploring the quantum effects is by studying the effective action, which can be obtained by using the standard covariant perturbation techniques. Covariant perturbative quantization of $2 \mathrm{~d}$ dilaton gravity theories has been studied by many authors [2, 3, 4, 5, 6, 7, 8, 9], but surprisingly, there has not been any systematic study of the effective action. In the context of $2 \mathrm{~d}$ sigma models, which are related to $2 \mathrm{~d}$ dilaton gravities, a one-loop contribution due to the scalar fields has been evaluated in [10]. In the case of the CGHS model [11], an incomplete one-loop effective action has been found from a combination of the path-integral measure and the $\beta$-function considerations [12, 13. Subsequently, further one-loop terms were found from the symmetry considerations 114, 15.

In order to give a systematic derivation of the one-loop CGHS effective action, and in order to do the same for a more realistic model of the spherically symmetric general relativity, we will study the one-loop effective action of a generic dilaton gravity theory coupled to conformal matter. The classical action of such a theory is given by

$$
S=\int d^{2} x \sqrt{\tilde{g}}\left[e^{-2 \Phi}\left(\tilde{R}+\alpha(\tilde{\nabla} \Phi)^{2}+U(\Phi)\right)-\frac{1}{2} \sum_{i}\left(\tilde{\nabla} f_{i}\right)^{2}\right],
$$

where $\tilde{g}, \tilde{R}$ and $\tilde{\nabla}$ stand for the determinant, the scalar curvature and the covariant derivative associated to the physical $2 \mathrm{~d}$ metric $\tilde{g}_{\mu \nu}, \alpha$ is a numerical constant and $i=1, \ldots, N$. $\Phi$ is the dilaton scalar field, while $f_{i}$ are the matter scalar fields. When $\alpha=4$ and $U(\Phi)=$ const. $=4 \lambda^{2}$ the action (1.1) is the CGHS model, while $\alpha=2$ and $U(\Phi)=e^{2 \Phi}$ gives the spherically symmetric general relativity coupled to null-dust (in units $G=1$, where $G$ is the Newton constant) [16], to which we refere as the SSND model. If the dilaton is redefined as $\phi=e^{-2 \Phi}$, and after an appropriate rescaling of the metric $\left(\tilde{g}_{\mu \nu}=e^{\alpha \Phi / 2} g_{\mu \nu}\right)$, the action (1.1) simplifies

$$
S=\int d^{2} x \sqrt{g}\left[\phi R+V(\phi)-\frac{1}{2} \sum_{i}\left(\nabla f_{i}\right)^{2}\right] .
$$

In this form the CGHS model is given by $V(\phi)=4 \lambda^{2}$, while the SSND model is given by $V(\phi)=1 / \sqrt{\phi}$. The form (1.2) is more convenient for the calculation of the effective action, and the only restriction on the dilaton potential will be that for large $\phi$ the potential $V$ will behave as $\phi^{-k}$, where $k \geq 0$. This ensures that in the weak-coupling limit $\phi \rightarrow \infty$ (which for the spherically symmetric general relativity 
means large radius $r$, since in that case $\phi=r^{2}$ [16]) we can ignore the contributions to the effective action proportional to $d^{n} V / d \phi^{n}$ or to $V^{n+1}$ for $n \geq 1$. In this limit we will calculate the complete one-loop effective action, which will include the loop contributions from the metric, the dilaton and the ghosts.

In section 2 we set up the background field method we will use for the calculation of the one-loop effective action. In section 3 we expand the results of section 2 to the first order in a perturbation of the background metric, since this simplifies the calculations. In section 4 we calculate the contribution to the effective action which is independent of $\phi$-derivatives. In section 5 we calculate the contribution for a flat background metric. In section 6 we combine the results of sectons 4 and 5 and give our final result. In section 7 we present our conclusions.

\section{The background field method}

In order to calculate the one-loop effective action we will use the background field method in a formulation given by Abbot [17]. The effective action $\Gamma\left[\phi_{0}\right]$ can be expressed as

$$
e^{i \Gamma\left[\phi_{0}\right]}=\int \mathcal{D} \phi e^{i\left(S(\phi)+\int d^{2} x J\left(\phi-\phi_{0}\right)\right)},
$$

where $\phi_{0}$ denotes a set of the classical background fields. $S$ is a sum of the classical action, the gauge-fixing action and the ghost action, so that the integration variable $\phi$ also includes the ghost fields. However, for the ghosts $J=0$ and $\phi_{0}=0$. The source $J$ is a function of $\phi_{0}$, which is determined from

$$
J=-\frac{\delta \Gamma}{\delta \phi_{0}}=-\frac{\delta S}{\delta \phi_{0}}+O(\hbar)
$$

One then splits $\phi$ as $\phi=\phi_{0}+\tilde{\phi}$ where $\tilde{\phi}$ is a new integration variable, called the quantum field. The path-integral (2.1) is then evaluated perturbatively, by Taylor expanding $S\left(\phi_{0}+\tilde{\phi}\right)$. For the one-loop approximation, one expands $S$ to the second order in $\tilde{\phi}$, while $J$ is expanded to the zeroth order in $\hbar$, so that

$$
e^{i \Gamma\left[\phi_{0}\right]} \approx e^{i S\left(\phi_{0}\right)} \int \mathcal{D} \tilde{\phi} e^{\frac{i}{2} S^{\prime \prime}\left(\phi_{0}\right) \tilde{\phi}^{2}}
$$

where " denotes the second functional derivative. Approximation (2.2) then yields the one-loop effective action formula

$$
\Gamma_{1}\left[\phi_{0}\right]=S\left(\phi_{0}\right)-\frac{1}{2 i} \operatorname{Tr}\left(\log S^{\prime \prime}\left(\phi_{0}\right)\right)
$$

In our case, we split the fields $\left\{g_{\mu \nu}, \phi, f\right\}$ into the classical background fields $\left\{g_{\mu \nu}, \phi, f_{0}\right\}$ and the quantum fields $\left\{h_{\mu \nu}, \hat{\phi}, f\right\}$ as

$$
g_{\mu \nu} \rightarrow g_{\mu \nu}+h_{\mu \nu} \quad, \quad \phi \rightarrow \phi+\hat{\phi} \quad, \quad f \rightarrow f_{0}+f \quad
$$


so that quadratic in quantum fields part of the action (1.2) is given by

$$
\begin{aligned}
S^{(2)}=\int d^{2} x \sqrt{g}[ & \frac{1}{4} \phi h^{\mu \nu} \square h_{\mu \nu}+\frac{1}{4} D_{\rho} \phi h D^{\rho} h-\frac{3}{4} D_{\beta} \phi h^{\rho \mu} D^{\beta} h^{\rho \mu} \\
& -D_{\nu} \phi D_{\rho} h h^{\rho \nu}-\frac{1}{2} \phi h^{\nu \beta} D_{\beta} D_{\mu} h_{\nu}^{\mu}+D_{\mu} \phi h^{\rho \mu} D^{\nu} h_{\rho \nu}+\frac{1}{2} D_{\rho} \phi h_{\beta}^{\mu} D_{\mu} h^{\rho \beta}+ \\
& +\frac{1}{2} \phi h D_{\mu} D_{\nu} h^{\mu \nu}+\phi h^{\mu \lambda} h_{\lambda}^{\nu} R_{\nu \mu}+\frac{1}{8} \phi R h^{2}-\frac{1}{4} \phi h \square h-\frac{1}{4} \phi R h_{\mu \nu} h^{\mu \nu} \\
& -\frac{1}{2} \phi h h^{\mu \nu} R_{\mu \nu}+\frac{1}{2} h \hat{\phi} R+\hat{\phi} D_{\mu} D_{\nu} h^{\mu \nu}-\hat{\phi} \square h-\hat{\phi} h^{\mu \nu} R_{\mu \nu} \\
& +\left(\frac{1}{8} h^{2}-\frac{1}{4} h^{\mu \nu} h_{\mu \nu}\right) V(\phi)+\frac{1}{2} V^{\prime \prime}(\phi) \hat{\phi}^{2}+\frac{1}{2} V^{\prime}(\phi) h \hat{\phi} \\
& -\frac{1}{2} g^{\mu \nu} \partial_{\mu} f \partial_{\nu} f-\frac{1}{2} \partial_{\mu} f_{0} \partial_{\nu} f_{0}\left(-\frac{1}{4} g^{\mu \nu} h_{\alpha \beta} h^{\alpha \beta}\right. \\
& \left.\left.-\frac{1}{2} h h^{\mu \nu}+h^{\mu \lambda} h_{\lambda}^{\nu}+\frac{1}{8} g^{\mu \nu} h^{2}\right)-\partial_{\mu} f \partial_{\nu} f_{0}\left(\frac{1}{2} h g^{\mu \nu}-h^{\mu \nu}\right)\right]
\end{aligned}
$$

The indices in (2.5) are lowered and raised by the classical metric $g_{\mu \nu}$, while $h=g^{\mu \nu} h_{\mu \nu}$ and $D_{\mu}$ is the covariant derivative associated to $g_{\mu \nu}$. $S^{(2)}$ has two type of gauge symmetries; classical and quantum. This ensures that after fixing of the quantum guage symmetry one still obtains a gauge-invariant effective action. We choose the gauge-fixing condition as

$$
\chi_{\mu}=D_{\lambda} h_{\mu}^{\lambda}-\frac{1}{2} D_{\mu} h-\frac{1}{\phi} D_{\mu} \hat{\phi}=0
$$

so that the gauge-fixing term in the action takes the form

$$
S_{G F}=-\frac{1}{2} \int d x \sqrt{g} \phi \chi_{\mu} \chi^{\mu}
$$

(2.7) is chosen such that the total action has a minimal structure, i.e. the second spacetime derivatives acting on the quantum fields appear only as $\square$. The ghost action will be analised later. By combining (2.5-7) we get

$$
\begin{aligned}
S_{\text {tot }}^{(2)}= & S^{(2)}+S_{G F} \\
= & \int d^{2} x \sqrt{g}\left[\frac{1}{4} \phi h^{\mu \nu} \square h_{\mu \nu}-\frac{1}{8} \phi h \square h+\frac{3}{8} D_{\rho} \phi h D^{\rho} h\right. \\
& -\frac{3}{4} D_{\beta} \phi h_{\rho \mu} D^{\beta} h^{\rho \mu}-\frac{1}{2} D_{\nu} \phi D_{\rho} h h^{\rho \nu}+D_{\mu} \phi h^{\rho \mu} D^{\nu} h_{\rho \nu}+\frac{1}{2} \phi h^{\mu \lambda} h_{\lambda}^{\nu} R_{\nu \mu} \\
& +\frac{1}{8} \phi R h^{2}-\frac{1}{2} \phi h h^{\mu \nu} R_{\mu \nu}+\frac{1}{2} h \hat{\phi} R-\frac{1}{2} \hat{\phi} \square h-\hat{\phi} h^{\mu \nu} R_{\mu \nu}+ \\
& \left(\frac{1}{8} h^{2}-\frac{1}{4} h_{\mu \nu} h^{\mu \nu}\right) V(\phi)+\frac{1}{2} V^{\prime \prime}(\phi) \hat{\phi}^{2}+\frac{1}{2} V^{\prime}(\phi) h \hat{\phi} \\
& -\frac{1}{2} g^{\mu \nu} \partial_{\mu} f \partial_{\nu} f+\partial_{\mu} f_{0} \partial_{\nu} f\left(h^{\mu \nu}-\frac{1}{2} h g^{\mu \nu}\right)-\frac{1}{2} \partial_{\mu} f_{0} \partial_{\nu} f_{0}\left(-\frac{1}{4} g^{\mu \nu} h_{\alpha \beta} h^{\alpha \beta}-\frac{1}{2} h h^{\mu \nu}\right.
\end{aligned}
$$




$$
\begin{aligned}
& \left.+h^{\mu \lambda} h_{\lambda}^{\nu}+\frac{1}{8} g^{\mu \nu} h^{2}\right)-\frac{1}{4} \phi R h_{\mu \nu} h^{\mu \nu}-\frac{1}{2} \phi h^{\nu \beta} h^{\mu \alpha} R_{\alpha \nu \beta \mu}-\frac{1}{2 \phi}(\partial \hat{\phi})^{2} \\
& \left.-\frac{1}{2} D_{\mu} D_{\rho} \phi h^{\mu \beta} h_{\beta}^{\rho}+\frac{1}{2} D_{\mu} D_{\nu} \phi h^{\mu \nu} h\right] .
\end{aligned}
$$

In order to remove $\phi$ from the kinetic terms for $h$ in (2.8) we rescale

$$
\sqrt{\phi} h_{\mu \nu} \rightarrow h_{\mu \nu} \quad, \quad \frac{\hat{\phi}}{\sqrt{\phi}} \rightarrow \hat{\phi} .
$$

This field redefinition does not change the path-integral measure, since the Jacobian of the transformation (2.9) is equal to one. This can be shown by using the dimensional regularization and the delta-function identity $\delta^{(2+\epsilon)}(0)=0 . \quad S_{t o t}^{(2)}$ then changes as follows

$$
\begin{aligned}
S_{\text {tot }}^{(2)}= & \int d^{2} x \sqrt{g}\left[\frac{1}{4} h^{\mu \nu} \square h_{\mu \nu}-\frac{1}{8} h \square h-D_{\rho} \Phi h D^{\rho} h\right. \\
+ & 2 D_{\beta} \Phi h^{\rho \mu} D^{\beta} h_{\rho \mu}+D_{\nu} \Phi D_{\rho} h h^{\rho \nu} \\
+ & \frac{1}{2} h^{\mu \lambda} h_{\lambda}^{\nu} R_{\nu \mu}+\frac{1}{8} h^{2} R-\frac{1}{4} R h_{\mu \nu} h^{\mu \nu} \\
- & \frac{1}{2} h h^{\mu \nu} R_{\mu \nu}+\frac{1}{2} h \hat{\phi} R-\hat{\phi} h^{\mu \nu} R_{\mu \nu}+\frac{1}{\phi}\left(\frac{1}{8} h^{2}-\frac{1}{4} h_{\mu \nu} h^{\mu \nu}\right) V(\phi)+\frac{1}{2} V^{\prime}(\phi) \hat{\phi} h \\
& +\frac{1}{2} V^{\prime \prime}(\phi) \phi \hat{\phi}^{2}-\frac{1}{2} g^{\mu \nu} \partial_{\mu} f \partial_{\nu} f+\frac{1}{\sqrt{\phi}} \partial_{\mu} f_{0} \partial_{\nu} f\left(h^{\mu \nu}-\frac{1}{2} h g^{\mu \nu}\right) \\
- & \frac{1}{2 \phi} \partial_{\mu} f_{0} \partial_{\nu} f_{0}\left(-\frac{1}{4} g^{\mu \nu} h_{\alpha \beta} h^{\alpha \beta}-\frac{1}{2} h h^{\mu \nu}+h^{\mu \lambda} h_{\lambda}^{\nu}+\frac{1}{8} g^{\mu \nu} h^{2}\right) \\
- & \frac{1}{2} h^{\nu \beta} h^{\mu \alpha} R_{\alpha \nu \beta \mu}+h_{\mu \nu} h^{\mu \nu}\left(\frac{1}{4} \square \Phi+\frac{7}{4}(\nabla \Phi)^{2}\right) \\
+ & h^{2}\left(-\frac{1}{8} \square \Phi-\frac{7}{8}(\nabla \Phi)^{2}\right)-2 D_{\lambda} \Phi h_{\mu}^{\lambda} D_{\nu} h^{\mu \nu} \\
- & 2 D_{\lambda} \Phi D_{\nu} \Phi h_{\mu}^{\lambda} h^{\mu \nu}+D_{\nu} \Phi D_{\rho} \Phi h h^{\rho \nu}-\frac{D^{\rho} D_{\mu} \phi}{2 \phi} h^{\mu \beta} h_{\rho \beta}+\frac{D_{\mu} D_{\lambda} \phi}{2 \phi} h h^{\lambda \mu} \\
+ & \frac{1}{2} D_{\mu} \hat{\phi} D^{\mu} h+\frac{1}{2} D_{\mu} \hat{\phi} h D^{\mu} \Phi-\frac{1}{2} \hat{\phi} D_{\mu} \Phi D^{\mu} h-\frac{1}{2} \hat{\phi} h(\nabla \Phi)^{2}-\frac{1}{2}(\nabla \hat{\phi})^{2} \\
& \left.+\hat{\phi} D_{\mu} \hat{\phi} D^{\mu} \Phi-\frac{1}{2} \hat{\phi}^{2}(\nabla \Phi)^{2}\right],
\end{aligned}
$$

where $\phi=e^{-2 \Phi}$. Instead of using $h_{\mu \nu}$ it is more convinient to use $\bar{h}_{\mu \nu}=\Pi_{\mu \nu}^{\rho \sigma} h_{\rho \sigma}=$ $h_{\mu \nu}-\frac{1}{D} h g_{\mu \nu}$ and $h$. We take $D=2+\epsilon$ as the dimension of the spacetime, since we are going to use the dimensional regularization [18], and

$$
\Pi_{\rho \sigma}^{\mu \nu}=\frac{1}{2}\left(\delta_{\rho}^{\mu} \delta_{\sigma}^{\nu}+\delta_{\sigma}^{\mu} \delta_{\rho}^{\nu}\right)-\frac{1}{D} g^{\mu \nu} g_{\rho \sigma}
$$

is the projector onto traceless states. We will also use the doubling trick of ref. [18, so that we relabel the quantum fields $\left\{\bar{h}^{\mu \nu}, h, \hat{\phi}, f\right\}$ in $(2.10)$ as $\left\{\bar{h}^{\prime} \mu \nu, h^{\prime}, \hat{\phi}^{\prime}, f^{\prime}\right\}$ 
and add to the action (2.10) the same action, but with the quantum fields relabeled as $\left\{\bar{h}^{\prime \prime \mu \nu}, h^{\prime \prime}, \hat{\phi}^{\prime \prime}, f^{\prime \prime}\right\}$. This allows us to work with the complex fields

$$
\bar{h}^{\mu \nu}=\frac{\bar{h}^{\prime \mu \nu}+i \bar{h}^{\prime \prime \mu \nu}}{\sqrt{2}}, \bar{h}^{* \mu \nu}=\frac{\bar{h}^{\prime \mu \nu}-i \bar{h}^{\prime \prime \mu \nu}}{\sqrt{2}}, \ldots,
$$

so that (2.10) can be rewritten as

$$
S_{\text {tot }}^{(2)}=\frac{1}{2} \int d x \sqrt{g}\left(\bar{h}^{* \mu \nu} h^{*} \hat{\phi}^{*} f^{*}\right) \hat{K}\left(I \square+\hat{K}^{-1} \hat{M}\right)\left(\begin{array}{c}
\bar{h}_{\rho \sigma} \\
h \\
\hat{\phi} \\
f
\end{array}\right)
$$

where $I=\operatorname{diag}\left(\Pi_{\rho \sigma}^{\mu \nu}, 1,1,1\right)$,

$$
\begin{aligned}
\hat{K} & =\left(\begin{array}{cccc}
\prod_{\mu \nu}^{\rho \sigma} & 0 & 0 & 0 \\
0 & -\frac{\epsilon}{2(2+\epsilon)} & -1 & 0 \\
0 & -1 & 2 & 0 \\
0 & 0 & 0 & 2
\end{array}\right), \\
\hat{K}^{-1} \hat{M} & =\left(\begin{array}{cccc}
\hat{V}_{\alpha \beta}^{\rho \sigma} & \hat{G}_{\alpha \beta} & \hat{H}_{\alpha \beta} & \hat{W}_{\alpha \beta} \\
\hat{M}^{\rho \sigma} & \hat{P} & \hat{Q} & \hat{X} \\
\hat{N}^{\rho \sigma} & \hat{L} & \hat{S} & \hat{E} \\
\hat{Y}^{\rho \sigma} & \hat{Z} & 0 & 0
\end{array}\right) .
\end{aligned}
$$

The matrix elements in (2.13) are defined as

$$
\begin{gathered}
\hat{V}_{\alpha \beta}^{\rho \sigma}=-\Pi_{\alpha \beta}^{\rho \sigma} R+\Pi_{\alpha \beta}^{\mu \nu}\left(R_{\mu}^{\rho} \delta_{\nu}^{\sigma}+R_{\mu}^{\sigma} \delta_{\nu}^{\rho}-R_{\mu \nu}^{\rho \sigma}-R_{\nu \mu}^{\rho \sigma}\right) \\
-3 \Pi_{\alpha \beta}^{\rho \sigma} \square \Phi+7 \Pi_{\alpha \beta}^{\rho \sigma}(\nabla \Phi)^{2}+2 D_{\lambda} \Phi\left(\Pi_{\alpha \beta}^{\sigma a} g^{\lambda \rho}+\Pi_{\alpha \beta}^{\rho a} g^{\lambda \sigma}\right. \\
\left.-\Pi_{\alpha \beta}^{\lambda \sigma} g^{a \sigma}-\Pi_{\alpha \beta}^{\lambda \rho} g^{a \sigma}\right) \vec{D}_{a}+4 \Pi_{\alpha \beta}^{\mu \nu}\left(\delta_{\mu}^{\rho} D^{\sigma} D_{\nu} \Phi\right. \\
\left.\quad-2 \delta_{\mu}^{\rho} D^{\sigma} \Phi D_{\nu} \Phi+\delta_{\mu}^{\sigma} D^{\rho} D_{\nu} \Phi-2 \delta_{\mu}^{\sigma} D^{\rho} \Phi D_{\nu} \Phi\right)+O\left(e^{2 \Phi}\right) \\
\hat{G}_{\alpha \beta}=\Pi_{\alpha \beta}^{\mu \nu}\left(\frac{2-\epsilon}{2+\epsilon} R_{\mu \nu}+2 \frac{3 \epsilon-2}{2+\epsilon} D_{\mu} \Phi D_{\nu} \Phi-2 \frac{\epsilon-2}{2+\epsilon} D_{\mu} D_{\nu} \Phi+2 D_{\mu} \Phi \vec{D}_{\nu}\right) \\
\hat{H}_{\alpha \beta}=-2 \Pi_{\alpha \beta}^{\mu \nu} R_{\mu \nu}, \\
\hat{M}^{\rho \sigma}=\frac{\hat{W}_{\alpha \beta}=2 \Pi_{\alpha \beta}^{\mu \nu} e^{\Phi} \partial_{\nu} f_{0} \vec{\partial}_{\mu}}{1+\epsilon} R^{\rho \sigma}-\frac{2+\epsilon}{1+\epsilon}\left(2 \frac{3 \epsilon-2}{2+\epsilon} D^{\rho} \Phi D^{\sigma} \Phi-2 \frac{\epsilon-2}{\epsilon+2} D^{\rho} D^{\sigma} \Phi+\left(\overleftarrow{\partial}^{\rho} D^{\sigma} \Phi+\overleftarrow{\partial^{\sigma}} D^{\rho} \Phi\right)\right) \\
\hat{P}=-\frac{\epsilon^{2}}{(1+\epsilon)(2+\epsilon)} R-\frac{2+\epsilon}{1+\epsilon}\left(-D^{\mu} \Phi \vec{\partial}_{\mu}\right. \\
\left.-\frac{-\epsilon^{2}+5 \epsilon+6}{(2+\epsilon)^{2}} \square \Phi+\frac{-4 \epsilon^{2}+3 \epsilon+6}{(2+\epsilon)^{2}}(\nabla \Phi)^{2}\right)+O\left(e^{2 \Phi}\right)
\end{gathered}
$$




$$
\begin{gathered}
\hat{Q}=-\frac{\epsilon}{1+\epsilon} R+\frac{2+\epsilon}{1+\epsilon}\left(2 \overleftarrow{\partial^{\mu}} D_{\mu} \Phi+2 \square \Phi+2(\nabla \Phi)^{2}\right)+O\left(e^{2 \Phi}\right) \\
\hat{X}=-e^{\Phi} \frac{\epsilon}{1+\epsilon} \partial_{\mu} f_{0} \vec{\partial}^{\mu} \\
\hat{N}^{\rho \sigma}=-\frac{1}{1+\epsilon} R^{\rho \sigma}-\frac{2+\epsilon}{2(1+\epsilon)}\left(2 \frac{3 \epsilon-2}{2+\epsilon} D^{\rho} \Phi D^{\sigma} \Phi-2 \frac{\epsilon-2}{\epsilon+2} D^{\rho} D^{\sigma} \Phi\right. \\
\left.+\left(\overleftarrow{\partial^{\rho}} D^{\sigma} \Phi+\overleftarrow{\partial^{\sigma}} D^{\rho} \Phi\right)\right) \\
\hat{L}=\frac{\epsilon}{2(2+\epsilon)(1+\epsilon)} R-\frac{\epsilon}{2(1+\epsilon)} D^{\mu} \Phi \vec{\partial}_{\mu}+\frac{2-\epsilon}{2+\epsilon} \square \Phi-\frac{-3 \epsilon^{2}+6 \epsilon+8}{2(1+\epsilon)(2+\epsilon)}(\nabla \Phi)^{2}+O\left(e^{2 \Phi}\right) \\
\hat{S}=-\frac{\epsilon}{2(1+\epsilon)} R+\frac{2+\epsilon}{1+\epsilon} \overleftarrow{\partial^{\mu}} D_{\mu} \Phi+\frac{1}{1+\epsilon} \square \Phi+\frac{1}{1+\epsilon}(\nabla \Phi)^{2}+O\left(e^{2 \Phi}\right) \\
\hat{E}=\frac{\epsilon}{2(1+\epsilon)} e^{\Phi} \partial^{\mu} f_{0} \vec{\partial}_{\mu} \\
\hat{Z}=-\frac{\epsilon}{2(2+\epsilon)} \overleftarrow{\hat{Y}^{\rho}} e^{\Phi} \partial_{\rho} f_{0}
\end{gathered}
$$

In the lowest-order weak-coupling approximation the matrix elements of (2.13) do not depend on the potential $V(\phi)$, because the terms $\frac{V(\phi)}{\phi}, V^{\prime}(\phi), V^{\prime \prime}(\phi) \phi$ are of $O\left(\phi^{-k-1}\right)$, and we can discard them . Therefore the leading-order weak-coupling approximation will not depend on the potential $V$.

After fixing of the quantum gauge symetries, we must introduce the corresponding ghost fields. Under the general coordinate transformation the quantum metric $h_{\mu \nu}$ and the field $\hat{\phi}$ transform as follows

$$
\begin{gathered}
\delta_{0} h_{\mu \nu}=-D_{\mu} \epsilon_{\nu}-D_{\nu} \epsilon_{\mu}+\left(D_{\nu} h_{\mu}^{\rho}+D_{\mu} h_{\nu}^{\rho}-D^{\rho} h_{\mu \nu}\right) \epsilon_{\rho} \\
\delta_{0} \hat{\phi}=-\epsilon^{\rho} \partial_{\rho}(\phi+\hat{\phi})
\end{gathered}
$$

The ghost action is then given by

$$
S_{g h}=\int d^{2} x \sqrt{g} \phi \bar{c}^{\mu}\left[-\square c_{\mu}-R_{\mu}^{\nu} c_{\nu}+\frac{1}{\phi} D_{\mu}\left(c^{\rho} \partial_{\rho} \phi\right)\right]
$$

where we write only the part of the ghost action which gives the contribution to the one-loop effective action. We can rescale

$$
\phi \bar{c}^{\mu} \rightarrow \bar{c}^{\mu}
$$

so that

$$
S_{g h}=\int d^{2} x \sqrt{g} \bar{c}^{\mu}\left[-\square c_{\mu}-R_{\mu}^{\nu} c_{\nu}+\frac{1}{\phi} D_{\mu}\left(c^{\rho} \partial_{\rho} \phi\right)\right]
$$




\section{Expansion around a flat metric}

The calculation of the one-loop effective action can be simplified by expanding the background metric around a flat metric $\eta_{\mu \nu}$ as

$$
g_{\mu \nu}=\eta_{\mu \nu}+\gamma_{\mu \nu}+O\left(\gamma^{2}\right) \text {. }
$$

After inserting (3.1) into (2.12) and (2.13), we get

$$
\sqrt{g}\left(I \square+\hat{K}^{-1} \hat{M}\right)=\operatorname{diag}\left(P_{\alpha \beta}^{\rho \sigma}, 1,1,1\right) \partial^{2}+K^{-1} M,
$$

where $\partial^{2}=\eta^{a b} \partial_{a} \partial_{b}, P_{\alpha \beta}^{\rho \sigma}$ is given in (3.8) and

$$
K^{-1} M=\left(\begin{array}{cccc}
\tilde{V}_{\alpha \beta}^{\rho \sigma} & G_{\alpha \beta} & H_{\alpha \beta} & W_{\alpha \beta} \\
M^{\rho \sigma} & P & Q & X \\
N^{\rho \sigma} & L & S & E \\
Y^{\rho \sigma} & Z & 0 & F
\end{array}\right) .
$$

The matrix elements in (3.3) which are relevant for our calculation are given by

$$
\begin{aligned}
\tilde{V}_{\alpha \beta}^{\rho \sigma}= & \overleftarrow{\partial}_{a} A_{\alpha \beta}^{a b \rho \sigma} \vec{\partial}_{b} \\
- & \left(\eta^{a b} P_{\epsilon \tau}^{\rho \sigma} S_{b \alpha \beta}^{\epsilon \tau}-2 \sqrt{g} \partial_{\lambda} \Phi\left(g^{\lambda \rho} \Pi_{\alpha \beta}^{\sigma a}+\Pi_{\alpha \beta}^{\rho a} g^{\lambda \sigma}-\Pi_{\alpha \beta}^{\lambda \sigma} g^{a \rho}-\Pi_{\alpha \beta}^{\lambda \rho} g^{a \sigma}\right)\right) \vec{\partial}_{a} \\
+ & \overleftarrow{\partial}_{a} \eta^{a b} P_{\alpha \beta}^{\epsilon \epsilon} S_{b \tau \epsilon}^{\rho \sigma}-\sqrt{g} \Pi_{\alpha \beta}^{\rho \sigma} R+\sqrt{g} \Pi_{\alpha \beta}^{\mu \nu}\left(R_{\mu}^{\rho} \delta_{\nu}^{\sigma}+R_{\mu}^{\sigma} \delta_{\nu}^{\rho}-R_{\mu \nu}^{\rho \sigma}-R_{\nu \mu}^{\rho \sigma}\right) \\
- & 3 \sqrt{g} \Pi_{\alpha \beta}^{\rho \sigma} \square \Phi+7 \sqrt{g} \Pi_{\alpha \beta}^{\rho \sigma}(\nabla \Phi)^{2}+ \\
+ & 4 \sqrt{g} \Pi_{\alpha \beta}^{\mu \nu}\left(\delta_{\mu}^{\rho} D^{\sigma} D_{\nu} \Phi-2 \delta_{\mu}^{\rho} D^{\sigma} \Phi D_{\nu} \Phi+\delta_{\mu}^{\sigma} D^{\rho} D_{\nu} \Phi-2 \delta_{\mu}^{\sigma} D^{\rho} \Phi D_{\nu} \Phi\right) \\
- & 2 \sqrt{g} \partial_{\lambda} \Phi\left(\Pi_{\alpha \beta}^{a \tau} g^{\lambda \epsilon}+\Pi_{\alpha \beta}^{\epsilon a} g^{\lambda \tau}\right. \\
- & \left.\Pi_{\alpha \beta}^{\lambda \epsilon} g^{a \tau}-\Pi_{\alpha \beta}^{\lambda \tau} g^{a \epsilon}\right) S_{a \epsilon \tau}^{\rho \sigma}+\eta^{a b} P_{\delta \eta}^{\epsilon \tau} S_{a \alpha \beta}^{\delta \eta} S_{b \epsilon \tau}^{\rho \sigma}+O\left(e^{2 \Phi}\right) \\
& P=\overleftarrow{\partial}_{a} \bar{\gamma}^{a b} \vec{\partial}_{b}-\frac{\epsilon^{2}}{(1+\epsilon)(2+\epsilon)} \sqrt{g} R-\frac{2+\epsilon}{1+\epsilon} \sqrt{g}\left(-D^{\mu} \Phi \vec{\partial}_{\mu}\right. \\
& \left.-\frac{-\epsilon^{2}+5 \epsilon+6}{(2+\epsilon)^{2}} \square \Phi+\frac{-4 \epsilon^{2}+3 \epsilon+6}{(2+\epsilon)^{2}}(\nabla \Phi)^{2}\right)+O\left(e^{2 \Phi}\right) \\
& S=\overleftarrow{\partial}_{a} \bar{\gamma}^{a b} \vec{\partial}_{b}-\frac{\epsilon}{2(1+\epsilon)} \sqrt{g} R \\
& +\sqrt{g}_{\frac{1}{1+\epsilon}}\left((2+\epsilon) \overleftarrow{\partial}^{\mu} D_{\mu} \Phi+\square \Phi+(\nabla \Phi)^{2}\right)+O\left(e^{2 \Phi}\right) \\
F= & \overleftarrow{\partial}{ }_{a}^{a b} \vec{\gamma}_{b},
\end{aligned}
$$


where

$$
\begin{gathered}
P_{\rho \sigma}^{\mu \nu}=\frac{1}{2}\left(\delta_{\rho}^{\mu} \delta_{\sigma}^{\nu}+\delta_{\sigma}^{\mu} \delta_{\rho}^{\nu}\right)-\frac{1}{D} \eta^{\mu \nu} \eta_{\rho \sigma} \\
S_{a \mu \nu}^{\rho \sigma}=2 \Gamma_{a(\mu}^{(\rho} \delta_{\nu)}^{\sigma)} \\
\bar{\gamma}^{\mu \nu}=\gamma^{\mu \nu}-\frac{1}{2} \gamma \eta^{\mu \nu} \\
A_{\alpha \beta}^{\rho \sigma a b}=P_{\alpha \beta}^{\rho \sigma} \bar{\gamma}^{a b}-\frac{1}{D} \eta^{a b}\left(\gamma^{\rho \sigma} \eta_{\alpha \beta}-\gamma_{\alpha \beta} \eta^{\rho \sigma}\right)
\end{gathered}
$$

and $\gamma=\gamma^{\mu \nu} \eta_{\mu \nu}$. In the case of the ghost action (2.31), expansion (3.1) yields

$$
\begin{aligned}
S_{g h}= & \int d^{2} x \bar{c}^{\mu}\left(\delta_{\mu}^{\nu} \partial^{2}+T_{\mu}^{\nu}\right) c_{\nu} \\
= & \int d x \bar{c}^{\mu}\left[\delta_{\mu}^{\nu} \partial^{2}+\delta_{\mu}^{\nu} \overleftarrow{\partial}_{a} \bar{\gamma}^{a b} \vec{\partial}_{b}-\Gamma_{a \mu}^{\nu} \eta^{a \sigma} \vec{\partial}_{\sigma}+\eta^{a b} \Gamma_{a \mu}^{\rho} \Gamma_{b \rho}^{\nu}\right. \\
& +\overleftarrow{\partial}_{\sigma} \eta^{a \sigma} \Gamma_{a \mu}^{\nu}+2 \partial_{\rho} \Phi\left(\eta^{\rho \nu}-\bar{\gamma}^{\rho \nu}\right) \vec{\partial}_{\mu}-2 \eta^{\rho \alpha} \Gamma_{\alpha \mu}^{\nu} \partial_{\rho} \Phi+\sqrt{g} R_{\mu}^{\nu} \\
& \left.-2 \delta^{\rho \nu} \Gamma_{\rho \mu}^{\alpha} \partial_{\alpha} \Phi-\left(\eta^{\rho \nu}-\bar{\gamma}^{\rho \nu}\right)\left(4 \partial_{\mu} \Phi \partial_{\rho} \Phi-2 \partial_{\mu} \partial_{\rho} \Phi\right)\right] c_{\nu} .
\end{aligned}
$$

After a suitable transformation of the complex fields in the path-integral (2.2), we get from (2.3) the following one-loop contribution the effective action

$$
\Gamma_{1}=\frac{i}{2} \operatorname{Tr} \log \left(1+K^{-1} M \frac{1}{\partial^{2}}\right)-i \operatorname{Tr} \log \left(1+T \frac{1}{\partial^{2}}\right)
$$

where $K^{-1} M$ and $T$ are defined by (3.3) and (3.9). After expanding the logarithm in (3.10), we obtain

$$
\begin{aligned}
\Gamma_{1}= & \frac{i}{2}\left[\left(\tilde{V}_{\alpha \beta}^{\rho \sigma} P_{\rho \sigma}^{\alpha \beta}+P+S+F\right) \frac{1}{\partial^{2}}-\frac{1}{2}\left(\tilde{V}_{\alpha \beta}^{\rho \sigma} \frac{1}{\partial^{2}} P_{\mu \nu}^{\alpha \beta} \tilde{V}_{\gamma \delta}^{\mu \nu} \frac{1}{\partial^{2}} P_{\rho \sigma}^{\gamma \delta}\right.\right. \\
& +2 G_{\alpha \beta} \frac{1}{\partial^{2}} P_{\mu \nu}^{\alpha \beta} M^{\mu \nu} \frac{1}{\partial^{2}}+2 H_{\alpha \beta} \frac{1}{\partial^{2}} P_{\mu \nu}^{\alpha \beta} N^{\mu \nu} \frac{1}{\partial^{2}}+ \\
& +2 Y_{\alpha \beta} \frac{1}{\partial^{2}} P_{\mu \nu}^{\alpha \beta} W^{\mu \nu} \frac{1}{\partial^{2}}+P \frac{1}{\partial^{2}} P \frac{1}{\partial^{2}}+S \frac{1}{\partial^{2}} S \frac{1}{\partial^{2}} \\
& \left.\left.+F \frac{1}{\partial^{2}} F \frac{1}{\partial^{2}}+2 Q \frac{1}{\partial^{2}} L \frac{1}{\partial^{2}}+2 X \frac{1}{\partial^{2}} Z \frac{1}{\partial^{2}}\right)\right] \\
& -i\left[T_{\nu}^{\mu} \delta_{\mu}^{\nu} \frac{1}{\partial^{2}}-\frac{1}{2} T_{\nu}^{\mu} \delta_{\rho}^{\nu} \frac{1}{\partial^{2}} T_{\sigma}^{\rho} \delta_{\mu}^{\sigma} \frac{1}{\partial^{2}}\right] .
\end{aligned}
$$




\section{Calculation of the effective action for $\phi=$ const.}

In this section we will compute the part of the effective action which is independent of $\partial \phi$, which can be done by taking $\phi$ to be a spacetime constant. Also, we will discard the terms proportional to $1 / \phi=e^{2 \Phi}$, since they give a sub-leading contribution in the weak-coupling approximation. First, we compute the terms in (3.11) which contain $\tilde{V}$. We denote the vertices with two, one and zero spacetime derivatives as $A, B$ and $C$, respectively. The contribution due to $\bar{h}_{\mu \nu}$ loops can be written as

$$
\begin{aligned}
\tilde{V} \frac{1}{\partial^{2}}-\frac{1}{2} \tilde{V} \frac{1}{\partial^{2}} \tilde{V} \frac{1}{\partial^{2}}= & (A+B+C) \frac{1}{\partial^{2}} \\
& -\frac{1}{2}\left(A \frac{1}{\partial^{2}} A \frac{1}{\partial^{2}}+B \frac{1}{\partial^{2}} B \frac{1}{\partial^{2}}+C \frac{1}{\partial^{2}} C \frac{1}{\partial^{2}}\right) \\
& -A \frac{1}{\partial^{2}} B \frac{1}{\partial^{2}}-A \frac{1}{\partial^{2}} C \frac{1}{\partial^{2}}-B \frac{1}{\partial^{2}} C \frac{1}{\partial^{2}} .
\end{aligned}
$$

Note that if we set $\Phi=$ const. in (3.4) and if we are not carefull when calculating the corresponding one-loop contribution to the effective action, we may obtain a result independent of $\Phi$, which is wrong. The term which requires a carefull consideration is $\int d x \sqrt{g} R \frac{1}{\square} \square \Phi$, and it gives a nonzero result for $\Phi=$ const., which is $\int d x \sqrt{g} R \Phi$.

Now, one can show that $A$ and $B$ diagrams are equal zero (see the Appendix). The diagram $C$ is given by (formula (A.5) of the Appendix)

$$
\begin{aligned}
C= & -\frac{i \pi^{\frac{D}{2}}}{(2 \pi)^{2}} \Gamma\left(-\frac{\epsilon}{2}\right) \int d x\left[\left(\frac{-D^{2}+D+2}{2}-\frac{4}{D}\right) \sqrt{g} R+\eta^{a b} P_{\delta \eta}^{\epsilon \tau} P_{\rho \sigma}^{\alpha \beta} S_{a \alpha \beta}^{\delta \eta} S_{b \epsilon \tau}^{\rho \sigma}\right] \\
& +4 \frac{i \pi^{\frac{D}{2}}}{(2 \pi)^{2}} \Gamma\left(-\frac{\epsilon}{2}\right) \frac{D+2}{2} \int d x \sqrt{g} R \Phi .
\end{aligned}
$$

Note that the last term in the first line of (4.2) is a non-covariant quantity. We will denote the diagram which contains a vertex $X$ and a vertex $Y$ as $X Y$, where $X, Y \in\{A, B, C, \ldots\}$. The $A A$ diagram is given by

$$
A A=\int d x d y A_{\alpha \beta}^{\rho \sigma a b}(x) A_{\gamma \delta}^{\mu \nu c d}(y) P_{\mu \nu}^{\alpha \beta} P_{\rho \sigma}^{\gamma \delta} \partial_{a}^{x} \partial_{d}^{y} G(x-y) \partial_{b}^{x} \partial_{c}^{y} G(y-x)
$$

where the Green's function $G(x-y)$ satisfies

$$
\square_{x} G(x-y)=\delta(x-y)
$$

By using (A.8), (4.3) becomes

$$
-\frac{i \pi^{\frac{D}{2}}}{(2 \pi)^{2}} \frac{D^{2}+D-2}{2} \Gamma\left(1-\frac{\epsilon}{2}\right) B\left(2+\frac{\epsilon}{2}, 2+\frac{\epsilon}{2}\right)\left(\int d x \sqrt{g} R \frac{1}{\square} R+\frac{4}{\epsilon\left(1+\frac{\epsilon}{2}\right)} \int d x \sqrt{g} R\right),
$$


where

$$
\int d x \sqrt{g} R \frac{1}{\square} R=\int d x d y \sqrt{g(x)} \sqrt{g(y)} R(x) G(x-y) R(y) .
$$

The $A B$ diagram is equal to zero. The $A C$ diagram is given by

$$
\int d x d y A_{\alpha \beta}^{\rho \sigma a b}(x) C_{\gamma \delta}^{\mu \nu}(y) \partial_{b}^{x} G(x-y) \partial_{a}^{x} G(y-x) P_{\mu \nu}^{\alpha \beta} P_{\rho \sigma}^{\gamma \delta} .
$$

If we use (A.10) and

$$
\begin{aligned}
A_{\alpha \beta}^{\rho \sigma a b} C_{\gamma \delta}^{\mu \nu} P_{\mu \nu}^{\alpha \beta} P_{\rho \sigma}^{\gamma \delta}= & \left(-\frac{D^{2}+D-2}{2}+\frac{D^{2}-4}{D}\right) \bar{\gamma}^{a b} R \\
& +\left(\frac{4}{D}-\frac{3}{2}\right)\left(D^{2}+D-2\right) \bar{\gamma}^{a b} \square \Phi,
\end{aligned}
$$

where $R=\partial_{a} \partial_{b} \gamma^{a b}-\square \gamma+O\left(\gamma^{2}\right)$, we get

$$
A C=2 \frac{i \pi^{\frac{D}{2}}}{(2 \pi)^{2}} \int d x \sqrt{g}\left(R \frac{1}{\square} R-R \Phi\right) .
$$

The $B B$ diagram is given by

$$
\begin{aligned}
B B=\int & d x d y\left[\left(-\eta^{a b} P_{\epsilon \tau}^{\rho \sigma} S_{b \alpha \beta}^{\epsilon \tau}(x)+4 D_{\lambda} \Phi\left(\Pi_{\alpha \beta}^{a \sigma} g^{\lambda \rho}-\Pi_{\alpha \beta}^{\lambda \sigma} g^{a \rho}\right)\right) \vec{\partial}_{a}\right. \\
& \left.+\overleftarrow{\partial^{a}} P_{\alpha \beta}^{\epsilon \tau} S_{a \epsilon \tau}^{\rho \sigma}(x)\right] G(x-y) P_{\mu \nu}^{\alpha \beta} P_{\rho \sigma}^{\gamma \delta}\left[\left(-P_{\chi \eta}^{\mu \nu} S_{c \gamma \delta}^{\chi \eta}(y) \eta^{c d}\right.\right. \\
& \left.\left.+4 D_{\kappa} \Phi(y)\left(\Pi_{\gamma \delta}^{\nu d} g^{\mu \kappa}-\Pi_{\gamma \delta}^{\nu \kappa} g^{d \mu}\right)\right) \vec{\partial}_{d}+\overleftarrow{\partial}^{c} P_{\gamma \delta}^{\eta \chi} S_{c \eta \chi}^{\mu \nu}(y)\right] G(y-x)
\end{aligned}
$$

From (A.13) and (4.10) we get

$$
\begin{aligned}
B B= & -4 \frac{i \pi^{\frac{D}{2}}}{(2 \pi)^{2}} B(1+\epsilon / 2,1+\epsilon / 2) \\
\cdot & {\left[\Gamma(1-\epsilon / 2) \int d x d y G(x-y) P_{\gamma \delta}^{\alpha \beta} P_{\rho \sigma}^{\mu \nu} \partial^{a} S_{a \mu \nu}^{\gamma \delta}(x) \partial^{c} S_{c \alpha \beta}^{\rho \sigma}(y)\right.} \\
& +\frac{1}{2} \Gamma\left(-\frac{\epsilon}{2}\right)\left(\eta^{a c} \int d x P_{\gamma \delta}^{\alpha \beta} P_{\rho \sigma}^{\mu \nu} S_{c \mu \nu}^{\gamma \delta}(x) S_{a \alpha \beta}^{\rho \sigma}(x)\right. \\
& \left.\left.-4 \int d x S_{a \alpha \beta}^{\epsilon \tau} P_{\mu \nu}^{\alpha \beta}\left(P_{\epsilon \tau}^{a \mu} g^{\lambda \nu}-P_{\epsilon \tau}^{\lambda \mu} g^{a \nu}\right) \partial_{\lambda} \Phi\right)\right],
\end{aligned}
$$

were we have taken into account that $\Phi$ is a spacetime constant. Since

$$
B(1+\epsilon / 2,1+\epsilon / 2)=1-\epsilon+O\left(\epsilon^{2}\right),
$$

(4.11) can be rewritten as

$$
\begin{aligned}
B B= & -4 \frac{i \pi^{\frac{D}{2}}}{(2 \pi)^{2}}\left\{\Gamma ( 1 - \frac { \epsilon } { 2 } ) \left[\int d x d y G(x-y) P_{\gamma \delta}^{\alpha \beta} P_{\rho \sigma}^{\mu \nu} \partial^{a} S_{a \mu \nu}^{\gamma \delta}(x) \partial^{c} S_{c \alpha \beta}^{\rho \sigma}(y)\right.\right. \\
& \left.+\eta^{a c} \int d x P_{\gamma \delta}^{\alpha \beta} P_{\rho \sigma}^{\mu \nu} S_{c \mu \nu}^{\gamma \delta}(x) S_{a \alpha \beta}^{\rho \sigma}(x)\right]+\frac{1}{2} \Gamma(-\epsilon / 2) \int d x\left(\eta^{a c} P_{\gamma \delta}^{\alpha \beta} P_{\rho \sigma}^{\mu \nu} S_{c \mu \nu}^{\gamma \delta} S_{a \alpha \beta}^{\rho \sigma}\right. \\
& \left.\left.-4(1-\epsilon) S_{a \alpha \beta}^{\epsilon \tau} P_{\mu \nu}^{\alpha \beta}\left(g^{\lambda \nu} P_{\epsilon \tau}^{a \mu}-P_{\epsilon \tau}^{\lambda \mu} g^{a \nu}\right) \partial_{\lambda} \Phi\right)\right\} .
\end{aligned}
$$


Covariantization of (4.13) gives

$$
\begin{aligned}
B B & =-8 \frac{i \pi^{\frac{D}{2}}}{(2 \pi)^{2}}\left(\int d x \sqrt{g} R \frac{1}{\square} R+4 \int d x \sqrt{g} R\right) \\
& -2 \frac{i \pi^{\frac{D}{2}}}{(2 \pi)^{2}} \Gamma\left(-\frac{\epsilon}{2}\right) \int d x\left[\eta^{a b} P_{\delta \eta}^{\epsilon \tau} P_{\rho \sigma}^{\alpha \beta} S_{a \alpha \beta}^{\delta \eta} S_{b \epsilon \tau}^{\rho \sigma}-2(1-\epsilon)(D+2) \sqrt{g} R \Phi\right] .
\end{aligned}
$$

By summing up the non-covariant terms in (4.14) and (4.2) we obtain a general coordinate invariant result. $B C$ and $C C$ diagrams are equal to zero.

There is also a contributions to the effective action from the term $P^{2}+S^{2}+N F^{2}$. By using (A.8) we get

$$
\begin{aligned}
P^{2}+S^{2}+N F^{2}= & -(N+2) \frac{i \pi^{\frac{D}{2}}}{(2 \pi)^{2}} \Gamma\left(1-\frac{\epsilon}{2}\right) B\left(2+\frac{\epsilon}{2}, 2+\frac{\epsilon}{2}\right)\left(\int d x \sqrt{g} R \frac{1}{\square} R\right. \\
& \left.+\frac{4}{\epsilon\left(1+\frac{\epsilon}{2}\right)} \int d x \sqrt{g} R\right)-2 \frac{i \pi^{\frac{D}{2}}}{(2 \pi)^{2}} \int d x \sqrt{g} R \Phi
\end{aligned}
$$

The other terms in (3.11) are either zero or of a sub-leading order in $e^{2 \Phi}$.

The contribution due to the ghost loops is given by the last line in (3.11)

$$
\begin{aligned}
T \frac{1}{\partial^{2}}-\frac{1}{2} T \frac{1}{\partial^{2}} T \frac{1}{\partial^{2}} & =(\bar{A}+\bar{B}+\bar{C}) \frac{1}{\partial^{2}} \\
& -\frac{1}{2}\left(\bar{A} \frac{1}{\partial^{2}} \bar{A} \frac{1}{\partial^{2}}+\bar{B} \frac{1}{\partial^{2}} \bar{B} \frac{1}{\partial^{2}}+\bar{C} \frac{1}{\partial^{2}} \bar{C} \frac{1}{\partial^{2}}\right) \\
& -\bar{A} \frac{1}{\partial^{2}} \bar{B} \frac{1}{\partial^{2}}-\bar{A} \frac{1}{\partial^{2}} \bar{C} \frac{1}{\partial^{2}}-\bar{B} \frac{1}{\partial^{2}} \bar{C} \frac{1}{\partial^{2}} .
\end{aligned}
$$

Again, we calculate only the diagrams $\bar{A}, \bar{B}, \bar{C}, \bar{A} \bar{A}, \bar{B} \bar{B}, \bar{C} \bar{C}, \bar{A} \bar{B}, \bar{A} \bar{C}, \bar{B} \bar{C}$, which apper in (4.16). The calculation is similar to the previous calculation, so that we give only the list of results

$$
\begin{gathered}
\bar{A}=\bar{B}=\bar{A} \bar{B}=\bar{B} \bar{C}=0 \\
\bar{C}=\int d x\left(\sqrt{g} R+\eta^{a b} \Gamma_{a \mu}^{\rho} \Gamma_{\nu \rho}^{b}-\partial_{\mu} \Phi \partial^{\mu} \gamma\right) G(0) \\
=-\frac{i \pi^{\frac{D}{2}}}{(2 \pi)^{2}} \Gamma\left(-\frac{\epsilon}{2}\right) \int d x\left(\sqrt{g} R+\eta^{a b} \Gamma_{a \nu}^{\rho} \Gamma_{b \rho}^{\nu}+\Phi \square \gamma\right), \\
\bar{A} \bar{A}=D \int d x d y \bar{\gamma}^{a b}(x) \bar{\gamma}^{c d}(y) \partial_{a}^{x} \partial_{d}^{y} G(y-x) \partial_{b}^{x} \partial_{c}^{y} G(x-y) \\
=-\frac{i \pi^{\frac{D}{2}}}{(2 \pi)^{2}} D \Gamma\left(1-\frac{\epsilon}{2}\right) B\left(2+\frac{\epsilon}{2}, 2+\frac{\epsilon}{2}\right)\left(\int R \frac{1}{\square} R+\frac{4}{\epsilon\left(1+\frac{\epsilon}{2}\right)} \int d x \sqrt{g} R\right),
\end{gathered}
$$




$$
\begin{gathered}
\bar{A} \bar{B}=\frac{i \pi^{\frac{D}{2}}}{(2 \pi)^{2}} \int d x \sqrt{g} R \Phi \\
\bar{A} \bar{C}=-\frac{i \pi^{\frac{D}{2}}}{(2 \pi)^{2}} \int d x \sqrt{g}\left(R \frac{1}{\square} R+2 R \Phi\right) \\
\bar{B} \bar{B}=-2 \frac{i \pi^{\frac{D}{2}}}{(2 \pi)^{2}}\left(\int d x \sqrt{g}\left(R \frac{1}{\square} R+4 R-2 R \Phi\right)+\Gamma\left(-\frac{\epsilon}{2}\right) \int d x\left(\Gamma_{c \mu}^{\nu} \Gamma_{d \nu}^{\mu} \eta^{c d}+\Phi \square \gamma\right)\right) .
\end{gathered}
$$

By collecting (4.1-2), (4.5), (4.9), (4.14-15) and (4.17-22), we get that the $\partial \phi$-independent part of $\Gamma_{1}$ is given by

$$
\Gamma_{1}^{(1)}=-\frac{N-24}{96 \pi} \int d x \sqrt{g} R \frac{1}{\square} R-\frac{N-24}{24 \pi \epsilon} \int d x \sqrt{g} R+\frac{5}{4 \pi} \int d x \sqrt{g} R \Phi .
$$

\section{Calculation of the effective action for $g_{\mu \nu}=\eta_{\mu \nu}$}

In this section we will compute $\Gamma_{1}$ in the special case of the flat background, i.e. when $g_{\mu \nu}=\eta_{\mu \nu}$. We start from (3.11) and by using (3.4) we get

$$
\begin{aligned}
\tilde{V}_{\alpha \beta}^{\rho \sigma} & =2 D_{\lambda} \Phi\left(\eta^{\rho \lambda} P_{\alpha \beta}^{a \sigma}+\eta^{\lambda \sigma} P_{\alpha \beta}^{a \rho}-\eta^{a \sigma} P_{\alpha \beta}^{\lambda \rho}-P_{\alpha \beta}^{\lambda \sigma} \eta^{a \rho}\right) \vec{\partial}_{a} \\
& -3 P_{\alpha \beta}^{\rho \sigma} \square \Phi+7 P_{\alpha \beta}^{\rho \sigma}(\nabla \Phi)^{2} \\
& +4 P_{\alpha \beta}^{\mu \nu}\left(\delta_{\mu}^{\rho} D^{\sigma} D_{\nu} \Phi-2 \delta_{\mu}^{\rho} D^{\sigma} \Phi D_{\nu} \Phi+\delta_{\mu}^{\sigma} D^{\rho} D_{\nu} \Phi-2 \delta_{\mu}^{\sigma} D^{\rho} \Phi D_{\nu} \Phi\right) \\
& +O\left(e^{2 \Phi}\right) .
\end{aligned}
$$

Since the terms with two derivatives do not appear in (5.1), our calculation will be simpler. Again, we will denote the terms with one derivate as $B$, and the terms without derivates as $C$. By using (A.5) we get

$$
C=-\frac{i \pi^{\frac{D}{2}}}{(2 \pi)^{2}}\left(D^{2}+D-2\right) \Gamma(-\epsilon / 2)\left(\frac{7}{2}-\frac{8}{D}\right) \int d x(\nabla \Phi)^{2}
$$

The BB diagram is given by

$$
\begin{aligned}
B B= & 4 \int d x d y D_{\lambda} \Phi(x)\left(\eta^{\rho \lambda} P_{\alpha \beta}^{a \sigma}+\eta^{\sigma \lambda} P_{\alpha \beta}^{a \rho}-\eta^{a \sigma} P_{\alpha \beta}^{\lambda \rho}-P_{\alpha \beta}^{\lambda \sigma} \eta^{a \rho}\right) \overrightarrow{\partial_{a}^{x}} G(x-y) \\
& D_{\kappa} \Phi(y)\left(\eta^{\mu \kappa} P_{\gamma \delta}^{b \nu}+\eta^{\nu \kappa} P_{\gamma \delta}^{b \mu}-\eta^{b \nu} P_{\gamma \delta}^{\kappa \mu}-P_{\gamma \delta}^{\kappa \nu} \eta^{b \mu}\right) \overrightarrow{\partial_{b}^{y}} G(y-x) P_{\mu \nu}^{\alpha \beta} P_{\rho \sigma}^{\gamma \delta} .
\end{aligned}
$$

By using (A.13) we obtain

$$
B B=4 \frac{i \pi^{\frac{D}{2}}}{(2 \pi)^{2}} \Gamma\left(-\frac{\epsilon}{2}\right) B\left(1+\frac{\epsilon}{2}, 1+\frac{\epsilon}{2}\right)\left(D^{2}+D-2\right) \int d x(\nabla \Phi)^{2} .
$$


The matrix elements $P$ and $S$ are given by the expresssions (3.5) and (3.6). After inserting $g_{\mu \nu}=\eta_{\mu \nu}$ we get

$$
\frac{1}{2}\left(P \frac{1}{\partial^{2}}-\frac{1}{2} P \frac{1}{\partial^{2}} P \frac{1}{\partial^{2}}\right)=-\frac{1}{2} \frac{i \pi^{\frac{D}{2}}}{(2 \pi)^{2}} \Gamma\left(-\frac{\epsilon}{2}\right)\left(\frac{4 \epsilon^{2}-3 \epsilon-6}{(1+\epsilon)(2+\epsilon)}-\frac{1}{4}\left(\frac{2+\epsilon}{1+\epsilon}\right)^{2}\right) \int d x(\nabla \Phi)^{2},
$$

and similarly

$$
\frac{1}{2}\left(S \frac{1}{\partial^{2}}-\frac{1}{2} S \frac{1}{\partial^{2}} S \frac{1}{\partial^{2}}\right)=-\frac{1}{2} \frac{i \pi^{\frac{D}{2}}}{(2 \pi)^{2}} \Gamma\left(-\frac{\epsilon}{2}\right)\left(\frac{1}{1+\epsilon}-\frac{1}{4}\left(\frac{2+\epsilon}{1+\epsilon}\right)^{2}\right) \int d x(\nabla \Phi)^{2}
$$

Next, we will calculate the $G M$ diagram. From (2.15) and (2.18) we get

$$
\begin{aligned}
G M & =\int d x d y G_{\alpha \beta}(x) G(x-y) M^{\rho \sigma}(y) P_{\rho \sigma}^{\alpha \beta} G(x-y) \\
& =-4 \frac{2+\epsilon}{1+\epsilon} \int d x d y P_{\rho \sigma}^{\mu \nu} D_{\mu} \Phi(x) D^{\sigma} \Phi(y) \partial_{\nu}^{x} \partial_{y}^{\rho} G(x-y) G(y-x),
\end{aligned}
$$

where we have descarded the terms which vanish after the integration. By using (A.13) we get

$$
\begin{aligned}
G M= & -4 \frac{i \pi^{\frac{D}{2}}}{(2 \pi)^{2}} \frac{2+\epsilon}{1+\epsilon}\left(\frac{1}{2} \Gamma\left(-\frac{\epsilon}{2}\right) B\left(1+\frac{\epsilon}{2}, 1+\frac{\epsilon}{2}\right) \frac{D^{2}+D-2}{2 D}-\Gamma(1-\epsilon / 2)(1-1 / D)\right) \\
& \cdot \int d x(\nabla \Phi)^{2}
\end{aligned}
$$

It is easy to see from (2.16) and (2.22) that the diagram $H N$ vanishes.

The diagram $Q L$ is given by

$$
Q L=-\epsilon \frac{2+\epsilon}{(1+\epsilon)^{2}} \int d x d y D_{\mu} \Phi(x) D_{\nu} \Phi(y) G(x-y) \partial_{x}^{\mu} \partial_{y}^{\nu} G(y-x) .
$$

From (A.13) we get

$$
Q L=2 \frac{i \pi^{\frac{D}{2}}}{(2 \pi)^{2}} \int d x(\nabla \Phi)^{2}
$$

The diagrams $W Y$ and $X Z$ are of the order $e^{2 \Phi}$, and we will descard them in the weak-coupling approximation.

The contribution from the ghost loops is determined by

$$
T_{\mu}^{\nu}=2 \partial^{\nu} \Phi \vec{\partial}_{\mu}-4 \partial_{\mu} \Phi \partial^{\nu} \Phi+2 \partial_{\mu} \partial^{\nu} \Phi,
$$

which is obtained from (3.9) by taking $g_{\mu \nu}=\eta_{\mu \nu}$. (5.11) gives only two diagrams differant from zero. These are $\bar{C}$ and $\bar{B} \bar{B}$. From (A.5) we get

$$
\bar{C}=\frac{4 i \pi^{\frac{D}{2}}}{(2 \pi)^{2}} \Gamma\left(-\frac{\epsilon}{2}\right) \int d x(\nabla \Phi)^{2}
$$


while (A.13) gives

$$
\bar{B} \bar{B}=-2 \frac{i \pi^{\frac{D}{2}}}{(2 \pi)^{2}} \Gamma\left(-\frac{\epsilon}{2}\right) \int(\nabla \Phi)^{2} .
$$

By colecting (3.11), (5.2), (5.4-6), (5.8), (5.10), (5.12-13) we get that the one loop correction to the effective action in the case of the flat background metric is given by

$$
\Gamma_{1}^{(2)}=-\frac{\pi^{\frac{D}{2}}}{2(2 \pi)^{2}}[-8 \Gamma(-\epsilon / 2)+23] \int d x(\nabla \Phi)^{2} .
$$

\section{The complete one-loop effective action}

In sections 4 and 5 we have found the one-loop effective action in two special cases: $\phi=$ const. and $g_{\mu \nu}=\eta_{\mu \nu}$. In this section we will compute the complete oneloop effective action by adding to $\Gamma_{1}{ }^{(1)}+\Gamma_{1}{ }^{(2)}$ the terms which vanish for $\phi=$ const. and $g_{\mu \nu}=\eta_{\mu \nu}$. It is easy to see from (3.4-7) and (2.14-27), that there is only one such term in the weak-coupling approximation. It is given by

$$
\int d x d y \sqrt{g(x)} \sqrt{g(y)} R(x) G(x-y)(\nabla \Phi(y))^{2},
$$

and our task is to determine the coefficient which multiplies it.

We start from the diagrams in (4.1). The term (6.1) appears only in the diagram $A C$, which is given by

$$
\begin{aligned}
A C=\int d x d y[ & \left.P_{\alpha \beta}^{\rho \sigma} \bar{\gamma}^{a b}(x)-\frac{1}{D}\left(\gamma^{\rho \sigma}(x) \eta_{\alpha \beta}-\gamma_{\alpha \beta}(x) \eta^{\rho \sigma}\right)\right]\left[7 P_{\gamma \delta}^{\mu \nu}(\nabla \Phi)^{2}\right. \\
& \left.-16 P_{\gamma \delta}^{\mu \epsilon} \partial^{\nu} \Phi(y) \partial_{\epsilon} \Phi(y)\right] \partial_{b}^{x} G(x-y) \partial_{a}^{x} G(y-x) P_{\mu \nu}^{\alpha \beta} P_{\rho \sigma}^{\gamma \delta} .
\end{aligned}
$$

In (6.2) we have written only the relevant terms of the C vertex. From (A. 10) we get

$$
A C=2 \frac{i \pi^{\frac{D}{2}}}{(2 \pi)^{2}} \int d x d y\left(\partial_{a} \partial_{b} \bar{\gamma}^{a b}(x)-\frac{1}{2} \square \gamma(x)\right)(\nabla \Phi(y))^{2} G(x-y)+O\left(\gamma^{2}\right) .
$$

By using $R=\partial_{a} \partial_{b} \bar{\gamma}^{a b}-\frac{1}{2} \square \gamma+O\left(\gamma^{2}\right)$ we can rewritte (6.3) in the form

$$
A C=2 \frac{i \pi^{\frac{D}{2}}}{(2 \pi)^{2}} \int d x d y \sqrt{g(x)} \sqrt{g(y)} R(x)(\nabla \Phi(y))^{2} G(x-y) .
$$

The term (6.1) appears in $P P$ and $S S$ diagrams, whose contribution is

$$
2 \frac{i \pi^{\frac{D}{2}}}{(2 \pi)^{2}} \int d x d y \sqrt{g(x)} \sqrt{g(y)} R(x)(\nabla \Phi(y))^{2} G(x-y) .
$$


(6.1) also appears in the diagrams with ghost loops. From (3.9) we see that the relevant part of the $\bar{A} \bar{C}$ digram is

$$
\bar{A} \bar{C}=-4 \int d x d y \bar{\gamma}^{a b}(x) \partial_{\mu} \Phi(y) \partial^{\mu} \Phi(y) \partial_{b}^{x} G(x-y) \partial_{a}^{x} G(y-x) .
$$

By using (A.10) we get

$$
\bar{A} \bar{C}=4 \frac{i \pi^{\frac{D}{2}}}{(2 \pi)^{2}} \int d x d y \sqrt{g(x)} \sqrt{g(y)} R(x)(\nabla \Phi(y))^{2} G(x-y) .
$$

After summing up (6.4), (6.5) and (6.7) we obtain

$$
\Gamma_{1}^{(3)}=-\frac{1}{2 \pi} \int d x d y \sqrt{g(x)} \sqrt{g(y)} R(x)(\nabla \Phi(y))^{2} G(x-y) .
$$

By collecting (4.23), (5.14) and (6.8) we get the bare effective action

$$
\begin{gathered}
\bar{\Gamma}_{1}=S-\frac{N-24}{96 \pi} \int d x \sqrt{g} R \frac{1}{\square} R-\frac{N-24}{24 \pi \epsilon} \int d x \sqrt{g} R-\frac{1}{2 \pi} \int d x R \frac{1}{\square}(\nabla \Phi)^{2} \\
-\frac{\pi^{\frac{\epsilon}{2}}}{8 \pi}[-8 \Gamma(-\epsilon / 2)+23] \int d x \sqrt{g}(\nabla \Phi)^{2}+\frac{5}{4 \pi} \int d x \sqrt{g} R \Phi+O\left(e^{2 \Phi}\right),
\end{gathered}
$$

where $S$ is the classical action (1.2). After making a modified minimal subtraction of the poles in (6.9) we get the renormalized one-loop effective action

$$
\begin{aligned}
\Gamma_{1}= & S-\frac{N-24}{96 \pi} \int d x \sqrt{g} R \frac{1}{\square} R-\frac{1}{\pi} \int d x \sqrt{g}\left(\frac{1}{2} R \frac{1}{\square}(\nabla \Phi)^{2}-\frac{5}{4} R \Phi+\frac{23}{8}(\nabla \Phi)^{2}\right) \\
& +O\left(e^{2 \Phi}\right) .
\end{aligned}
$$

The expression (6.10) is our final result. By going back to the original form of the action via $\tilde{g}_{\mu \nu}=e^{\alpha \Phi / 2} g_{\mu \nu}$ we get

$$
\begin{aligned}
\Gamma_{1}= & S-\frac{N-24}{96 \pi}\left(\int d x \sqrt{\tilde{g}}\left(\tilde{R} \frac{1}{\square} \tilde{R}-\frac{\alpha^{2}}{4}(\tilde{\nabla} \Phi)^{2}+\alpha \tilde{R} \Phi\right)\right)+\frac{5 \alpha}{4 \pi} \int d x \sqrt{\tilde{g}} \tilde{R} \Phi \\
& -\frac{1}{2 \pi} \int d x \sqrt{\tilde{g}} \tilde{R} \frac{1}{\square}(\tilde{\nabla} \Phi)^{2}-\frac{\alpha}{4 \pi} \int d x \sqrt{\tilde{g}} \Phi(\tilde{\nabla} \Phi)^{2}-\frac{5 \alpha+23}{8 \pi} \int d x \sqrt{\tilde{g}}(\tilde{\nabla} \Phi)^{2} \\
& +O\left(e^{2 \Phi}\right),
\end{aligned}
$$

where now $\mathrm{S}$ is given by (1.1). In the case of large $N,(6.11)$ gives

$$
\Gamma_{1}=S-\frac{N}{96 \pi}\left(\int d x \sqrt{\tilde{g}} \tilde{R} \frac{1}{\square} \tilde{R}-\frac{\alpha^{2}}{4} \int d x \sqrt{\tilde{g}}(\tilde{\nabla} \Phi)^{2}+\alpha \int d x \sqrt{\tilde{g}} \tilde{R} \Phi\right)+O(1 / N) .
$$

In the case of the CGHS model, this is the action proposed in [15]. This action also coincides with the one-loop action obtained from of the operator quantization of the CGHS model [19]. 


\section{Conclusions}

The results (6.10-11) imply that the leading-order weak-coupling contribution to the effective action is independent of the potential $V$. The model dependence can be seen in the form (6.11), where it comes from the dilaton kinetic term coefficient $\alpha$ of the original action (1.1). The large- $N$ one-loop corrections are similar to the BPP case [15], but with the model-dependent coefficients. Only the Polyakov-Liouville term has a model-independent coefficient, which is not surprising, since its origin is from the integration of the matter fields, whose Lagrangean is model independent. Note that we have obtained the coefficient $N-24$ multiplying the Polyakov-Liouville term, which can be understood as $c=N+2-26$ conformal anomaly, where $N$ is the contribution from the matter fields, 2 is the contribution from the dilaton and the conformal factor and -26 is the ghost contribution.

It is interesting that the weak-coupling approximation does not coincide with the large-N approximation, given that both approximations are semiclassical. This is not that surprising, since the weak-coupling approximation is a more general one, because besides the matter loops it also includes the graviton and the dilaton loops. In the context of spherically symmetric general relativity this means that the large black hole mass approximation (large $N$ ) is included in the large radius approximation. An intriguing feature is that the operator quantization of the CGHS model gives the large-N result (6.12) as the exact one-loop result [19], while the covariant perturbation theory seems to give a non-zero contribution for the lower orders in $N$. It is possible that a resummation of the lower-order in $N$ terms exist, such that it gives zero. The second possibility is that these two methods give results which are not equivalent when $N$ is not large. It would be interesting to explore this issue for the case of the spherically symmetric general relativity as well as for the case of the CGHS two-loop approximation [20].

\section{APPENDIX A}

In this appendix we will compute diagrams which we need for evaluating $\Gamma_{1}$. We will use the dimensional regularization with $D=2+\epsilon$. In our case the relevant lagrangean can be written in the form

$$
\mathcal{L}=\phi^{*}\left(\square+\overleftarrow{\partial}_{a} A^{a b}(x) \vec{\partial}_{b}+B_{1}^{b}(x) \vec{\partial}_{b}+\overleftarrow{\partial}_{b} B_{2}^{b}(x)+C(x)\right) \phi
$$

where now $\phi$ denotes the set of relevant fields. 
The $A$ diagram is given by

$$
A=\lim _{x^{\prime} \rightarrow x} \int d x A^{a b}(x) \partial_{a} \partial_{b}^{\prime} G\left(x-x^{\prime}\right)=-\int d x A^{a b}(x) \int \frac{d^{2} k}{(2 \pi)^{2}} \frac{k_{a} k_{b}}{k^{2}} .
$$

(A.2) is infrared divergent and we must regularize it. We use a modified minimal subtraction scheme [21], where a counterterm is added to the propagator

$$
\frac{1}{k^{2}} \rightarrow \frac{1}{k^{2}}-\frac{2 i \pi^{\frac{D}{2}}}{\epsilon} \delta(k) \Gamma(1-\epsilon / 2)
$$

By inserting (A.3) into (A.2) we get $A=0$. Similarly, we get $B=0$. The diagram $C$ is given by

$$
\int d x C(x) G(0)=-\int d x C(x) \int \frac{d^{2} k}{(2 \pi)^{2}}\left(\frac{1}{k^{2}}-\frac{2 \pi^{\frac{D}{2}} i}{\epsilon} \delta(k) \Gamma(1-\epsilon / 2)\right) .
$$

The first term in (A.4) is equal to zero, so that

$$
C=-\frac{i \pi^{\frac{D}{2}}}{(2 \pi)^{2}} \Gamma(-\epsilon / 2) \int d x C(x) .
$$

Therefore $C$ is a UV divergent constant.

The $A A$ diagram is given by

$$
A A=\int d x d y A^{a b}(x) A^{c d}(y) \partial_{a}^{x} \partial_{d}^{y} G(x-y) \partial_{b}^{x} \partial_{c}^{y} G(y-x),
$$

which is UV divergent. After a suitable Fourier transformation we arrive at

$$
\begin{aligned}
A A= & \frac{1}{4} \int \frac{d p_{1}}{(2 \pi)^{4}} A^{a b}\left(p_{1}\right) A^{c d}\left(-p_{1}\right) \\
& \cdot \int d k \frac{\left[k_{a}\left(p_{1}+k\right)_{b}+k_{b}\left(p_{1}+k\right)_{a}\right]\left[k_{d}\left(p_{1}+k\right)_{c}+k_{c}\left(p_{1}+k\right)_{d}\right]}{k^{2}\left(k+p_{1}\right)^{2}} .
\end{aligned}
$$

This gives

$$
\begin{gathered}
A A=\frac{i \pi^{\frac{D}{2}}}{(2 \pi)^{2}} \Gamma(1-\epsilon / 2) B(2+\epsilon / 2,2+\epsilon / 2)\left[-\int d x d y \partial_{a} \partial_{b} A^{a b}(x) G(x-y) \partial_{c} \partial_{d} A^{c d}(y)\right. \\
\left.+\int d x\left(-\frac{1}{2 \epsilon\left(1+\frac{\epsilon}{2}\right)}\left[A_{a}^{a} \square A_{b}^{b}+2 A^{a b} \square A_{a b}\right]+\frac{1}{\epsilon}\left[3 \partial_{b} A^{a b} \partial_{d} A_{a}^{d}-2 A_{a}^{a} \partial_{c} \partial_{b} A^{b c}\right]\right)\right] . \quad \text { (A.8) }
\end{gathered}
$$

The $A B_{1}$ diagram is given by

$$
\begin{aligned}
A B_{1}= & \int d x d y A^{a b}(x) B_{1}^{c}(y) \partial_{b}^{x} G(x-y) \partial_{c}^{y} \partial_{a}^{x} G(y-x) \\
= & \frac{i \pi^{\frac{D}{2}}}{(2 \pi)^{2}} B(2+\epsilon / 2,1+\epsilon / 2)\left[\Gamma(1-\epsilon / 2) \int d x d y \partial_{a} \partial_{b} A^{a b}(x) G(x-y) \partial_{c} B_{1}^{c}(y)\right. \\
& \left.-\frac{1}{2} \Gamma(-\epsilon / 2) \int d x A_{a}^{a} \partial_{c} B_{1}^{c}\right] .
\end{aligned}
$$


The $A B_{2}$ diagram is the same as the $A B_{1}$ diagram, due to the symmetry of $A^{a b}$.

The $A C$ diagram is given by

$$
\begin{aligned}
A C= & \int d x d y A^{a b}(x) C(y) \partial_{b}^{x} G(x-y) \partial_{a}^{x} G(y-x) \\
= & \frac{i \pi^{\frac{D}{2}}}{(2 \pi)^{2}} B(1+\epsilon / 2,1+\epsilon / 2)\left[-\Gamma(1-\epsilon / 2) \int d x d y \partial_{a} \partial_{b} A^{a b}(x) G(x-y) C(y)\right. \\
& \left.+\frac{1}{2} \Gamma(-\epsilon / 2) \int d x A_{a}^{a} C\right] .
\end{aligned}
$$

The $B B$ diagram is given by

$$
\begin{aligned}
& B B=\int d x d y[\left(B_{1}^{a}(x) B_{1}^{b}(y)+B_{2}^{a}(x) B_{2}^{b}(y)\right) \partial_{a}^{x} G(x-y) \partial_{b}^{y} G(y-x) \\
&\left.+\left(B_{2}^{a}(x) B_{1}^{b}(y)+B_{1}^{a}(x) B_{2}^{b}(y)\right) \partial_{b}^{y} \partial_{a}^{x} G(x-y) G(y-x)\right] .
\end{aligned}
$$

If we make a Fourier transformation as before, we obtain

$$
\begin{aligned}
B B & =\frac{i \pi^{\frac{D}{2}}}{(2 \pi)^{2}}\left[-\Gamma(1-\epsilon / 2) B(1+\epsilon / 2,1+\epsilon / 2) \int d x d y\left(\partial_{a} B_{1}^{a}(x) \partial_{b} B_{1}^{b}(y)\right.\right. \\
& \left.+\partial_{a} B_{2}^{a}(x) \partial_{b} B_{2}^{b}(y)-2 \partial_{a} B_{1}^{a}(x) \partial_{b} B_{2}^{b}(y)\right) G(x-y) \\
& +\frac{1}{2} \Gamma(-\epsilon / 2) B(1+\epsilon / 2,1+\epsilon / 2) \eta_{a b} \int d x\left(2 B_{2}^{a} B_{1}^{b}-B_{1}^{a} B_{1}^{b}-B_{2}^{a} B_{2}^{b}\right) \\
& \left.-2 \Gamma(1-\epsilon / 2) B(\epsilon / 2,1+\epsilon / 2) \int d x d y\left(\partial_{a} B_{1}^{a}(x) \partial_{b} B_{2}^{b}(y)\right) G(x-y)\right] .
\end{aligned}
$$

From (A.12) we see that the $B B$ diagram is both IR and UV divergent. As before, we remove the IR divergence by the regularization (A.3). By using $B(\epsilon / 2,1+\epsilon / 2)=$ $2 / \epsilon+O(\epsilon)$ we obtain

$$
\begin{aligned}
B B & =\frac{i \pi^{\frac{D}{2}}}{(2 \pi)^{2}}\left[-\Gamma(1-\epsilon / 2) B(1+\epsilon / 2,1+\epsilon / 2) \int d x d y\left(\partial_{a} B_{1}^{a}(x) \partial_{b} B_{1}^{b}(y)\right.\right. \\
& \left.+\partial_{a} B_{2}^{a}(x) \partial_{b} B_{2}^{b}(y)-2 \partial_{a} B_{1}^{a}(x) \partial_{b} B_{2}^{b}(y)\right) G(x-y) \\
& \left.+\frac{1}{2} \Gamma(-\epsilon / 2) B(1+\epsilon / 2,1+\epsilon / 2) \eta_{a b} \int d x\left(2 B_{2}^{a} B_{1}^{b}-B_{1}^{a} B_{1}^{b}-B_{2}^{a} B_{2}^{b}\right)\right] .
\end{aligned}
$$

The diagrams $B C$ and $C C$ are only IR divergent. After using (A.3) we get that they are equal to zero. In our calculations we have ignored the term $\ln \frac{p^{2}}{\mu^{2}}$, where $\mu$ has the dimension of mass.

\section{References}

[1] A. Strominger, Les Houches Lectures on Black Holes, Santa Barbara preprint (1995), hep-th/9501071 
[2] R. Gastmans, R. Kallosh and C. Truffin, Nucl. Phys. B 133 (1987) 417

[3] H. Kawai and M. Ninomiya, Nucl. Phys. B 336 (1990) 115

[4] S. D. Odintsov and I. L. Shapiro, Class. Quant. Grav.8 (1991) L57

[5] S. D. Odintsov and I. L. Shapiro, Phys. Lett. 263B (1991) 183

[6] I. L. Buchbinder, S. D. Odintsov and I. L. Shapiro, Effective Action in Quantum Gravity, IOP Publishing, Bristol and Philadephia (1992)

[7] H. Kawai, Y. Kitazawa and M. Ninomiya, Nucl. Phys. B 393 (1993) 280; Nucl. Phys. B 404 (1993) 684

[8] S. Kojima, N. Sakai and Y. Tanii, Nucl. Phys. B 426 (1994) 223

[9] I. L. Buchbinder, I. L. Shapiro and A. G. Sibiryakov, Nucl. Phys. B 445 (1995) 109

[10] C. M. Hull and P. K. Townsend, Nucl. Phys. B 274 (1986) 349

[11] C. G. Callan, S.B Giddings, J. A. Harvey and A. Strominger, Phys. Rev. D45 (1992) R1005

[12] S. P. de Alwis, Phys. Lett. 289B (1992) 278

[13] S. B. Giddings and A. Strominger Phys. Rev. D 47 (1993) 2454

[14] J. G. Russo, L. Susskind and L. Thorlacius, Phys. Rev. D 46 (1992) 3444; Phys. Rev. D 47 (1993) 533

[15] S. Bose, L. Parker and Y. Peleg, Phys. Rev. D 52 (1995) 3512

[16] J. Cruz, A. Miković and J. Navarro-Salas, A quantum model of Schwarzschild black hole evaporation, University of Valencia preprint FTUV-96-79 (1996)

[17] L.F. Abbot, Nucl. Phys. B 185 (1981) 189

[18] G. 't Hooft and M. Veltman, Ann. Inst. H. Poincare 20, 1 (1974) 69

[19] A. Miković, Class. Quant. Grav.13 (1996) 209

[20] A. Miković and V. Radovanović, Nucl. Phys. B 481 (1996) 719

[21] B. de Wit, M. T. Grisaru and P. van Nieuwenhizen, Nucl. Phys. B 408 (1993) 299 